\title{
A RATIONAL APPROACH FOR PREDICTING THE MINIMUM COMPOSITION OF ANTI-PARASITE SUB-UNIT VACCINES: A MULTIPLE TARGET VACCINE HYPOTHESIS.
}

\section{Vincent P.K.Titanji, ${ }^{1,2}$ Jerome Nyhalah Dinga, ${ }^{2}$ Raymond Babila N yasa ${ }^{2}$}

${ }^{1}$ Faculty of Science, Engineering and Technology, Cameroon Christian University Institute, Bali, North West Region, Cameroon.

${ }^{2}$ Biotechnology Unit, Faculty of Science, University of Buea, Box 63 Buea, South West Region, Cameroon. *Address for correspondence. Email: vpk.titanji@yahoo.com

\begin{abstract}
ABST RACT
The present article introduces a new concept, termed a multiple vaccine target hypothesis (MVTH) for determining the minimum number of vaccine targets (epitopes/ antigens) necessary to construct a highly efficacious (greater than 90\%) anti-parasite vaccine. D rawing inspiration from the Metabolic Control Analysis (MCA) and immunological reasoning it is proposed that for a multi-stage parasite an effective anti-parasite vaccine will necessarily act on more than one stage of the parasite in the definitive host. It is argued that the minimum number of sub-unit targets for a highly efficacious anti-parasite vaccine will vary from one parasite to the other and will be equal to twice the number of major parasite stages occurring in the human host. Q uantitatively stated, $\mathrm{C}_{\mathrm{v}}=2 \mathrm{n}$ where $\mathrm{C}_{\mathrm{v}}$ the number of required targets is, and $n$ represents the number of major parasite stages in the definitive host. Conditions when the value of $\mathrm{C}_{\mathrm{v}}$ may deviate from what is predicted by the formula given above are discussed. Extensive literature search on malaria, onchocerciasis and schistosomiasis sub-unit vaccine development suggests that subunit vaccines constructed from single components and directed to just one parasite stage are less efficacious that multivariate sub-unit vaccines, and obviously whole parasite vaccines. MVTH therefore provides a rational framework for constituting a sub-unit anti-parasite vaccine.
\end{abstract}

Keywords: Vaccine, malaria, O nchocerca volvulus, schistosomiasis, parasite, equation

\section{RESUME}

L'actuel article introduit une nouvelle méthode d'estimation de nombre minimum de composantes dans un vaccin moléculaire contre les parasites humains. Basé sur les concepts de contrôle des voies métaboliques et compte tenu des principes immunologues, il est proposé que le nombre de composantes requises pour un vaccin moléculaire efficace contre un parasite, $\mathrm{C}_{\mathrm{v}}=2 \mathrm{n}$, ou $\mathrm{n}=$ le nombre stades parasitaires dans l'hôte immunocompétent. Les conditions qui permettent de dévier de cette équation sont discutées. Les données préliminaire de notre laboratoire ainsi que les publications parues sur le développement des vaccins contre le paludisme, l'onchocercose et les bilharzioses humaines sont citées en appui de l’hypothèse sus-évoquée.

Mots clés: Vaccin, paludisme, l’onchocercose, bilharziose, parasite, équation

(C) The Authors. This work is licensed under the Creative Commons Attribution 4.0 International Licence. 


\section{INTRODUCTION}

The major parasitic infections of humans including malaria, schistosomiasis and river blindness can be controlled or even eliminated if effective vaccines are developed and used in combination with other strategies like vector control, chemotherapy and environmental remediation. However, it has so far proved difficult to produce a widely effective vaccine against any major human parasite despite evidence from a variety of animal models and clinical trials that this is feasible (Bruder et al, 2010; Makepeace et al, 2015; Tebeje et al, 2016). Many researchers taking advantage of the power of recombinant DNA technology have opted for the production of sub-unit vaccines (D raper et al; 2010). The basic assumption for the design of anti-parasite sub-unit vaccines is that there exists a single target in the parasite, which if blocked, would completely interrupt the parasite's life cycle thereby eliminating it. This hypothesis is similar to the rate-limiting hypothesis which held that there existed in a metabolic pathway an enzymatic step whose rate determined the flux or rate of the entire pathway. The rate-limiting hypothesis has since been debunked following the development of Metabolic Control Analysis (MCA) and the confirmation of its predictions by numerous experimental results (Fell,1992 \& 1997)

D rawing inspiration from the Metabolic Control Analysis it is proposed that:

Postulate I: The inhibition by vaccination of just one target (epitope/ antigen) on a parasite is insufficient to block the entire life cycle flux i.e. production of the transmissible stage, unless the vaccine acts like a drug or strong inhibitor or the target appears repeatedly on more than one parasite stage.

Postulate II: An effective sub-unit vaccine should target not just one parasite stage, but multiple if not all the main stages occurring within the definitive host.
Furthermore, based on the assumption that both the humoral and cellular arms of the immune response are needed for protective immunity it is proposed that:

Postulate III: At least one target epitope/ antigen respectively for the B and T-cells from each of the main stages of the parasite developing within the immunocompetent host will be required for an effective sub-unit vaccine.

The three postulates formulated above together constitute what is herein termed the multiple vaccine target hypothesis (MVTH). The aim of this report is to present this new approach for predicting the sub-unit composition of a multistage parasite and to cite supporting evidence from vaccine studies against malaria, onchocerciasis and schistosomiasis, which are human infections with devastating public health consequences (WHO, 2016)

\section{The analogy between metabolic pathways and a parasite's life cycle}

2.1 Main Conclusions of the MCA relevant to a parasite's lifecycle

A segment of the life cycle of a parasite in the host can be compared to a metabolic pathway since both comprise of multiple sequential time-bound steps driven by enzymatic processes. Conclusions drawn from MCA can possibly be applied to the life cycle segment occurring within the immunocompetent host. Consider the metabolic pathway

$A \stackrel{e_{1}}{\longrightarrow} B \stackrel{e_{2}}{\longrightarrow} C \stackrel{e_{3}}{\longrightarrow} D$

Let A be the source and D the sink; B, C, intermediates and $e_{1}, e_{2}, e_{3} \ldots$ the enzymes that catalyze the respective steps in the reactions. According to the MCA, there is no rate limiting enzyme which determines the flux. In order to inhibit the pathway several enzymes, particularly those which are far from equilibrium must be inhibited simultaneously. Conversely, in order to increase the flux through the pathway several or all of the enzymes must be activated. Thus in 
order to change the flux, several or all of the enzymes have to be affected (Fell, 1992; Liao and Delgado, 1993).

At dynamic equilibrium,

$$
\begin{aligned}
& \text { Box } 1 \\
& \text { The } \operatorname{Flux}(J)=\frac{d D}{d t} \\
& \text { The flux control coefficient: } C_{e_{2}}^{J}=\frac{\partial \ln e_{3}}{\partial \ln e_{2}}=\frac{\partial \ln V_{\max e_{3}}}{\partial \ln V_{\max e_{2}}} \text {. } \\
& \text { The concentration control Coefficient : } C_{e_{2}}^{B}=\frac{\partial B}{\partial e_{2}} \cdot \frac{V_{e_{2}}}{B}=\frac{\partial \ln B}{\partial \ln V_{e_{2}}} \\
& \text { The elasticity coefficient : } \varepsilon_{B}^{e_{2}}=\frac{\partial V_{e_{2}}}{\partial B} \cdot \frac{B}{V_{e_{2}}}=\frac{\partial \ln \left|V_{e_{2}}\right|}{\partial \ln B} \\
& \text { The response coefficient to a factor }(p): \boldsymbol{R}_{p}^{J}=\frac{\partial J_{e_{2}}}{\partial p} \cdot \frac{p}{\partial J_{e_{2}}}=C_{e_{1}}^{J_{e_{2}}} \cdot \boldsymbol{\varepsilon}_{p}^{e_{1}} \\
& \text { The summation theorem: } \sum_{i=1}^{n} C_{i}^{J}=1 \\
& \text { The connectivity theorem : } \sum_{i=1}^{n} C_{i}^{J} \varepsilon_{s}^{L}=0 \\
& \text { where } s=\text { concentration of metabolites } A, B, C, D \text {, in (1) } \\
& V_{\max e_{3}}=V_{e_{3} ;} V_{\max e_{2}}=V_{e_{2} ;} \text { etc. }
\end{aligned}
$$

The MCA defines the Control, Elasticity and Response coefficients, which describe quantitatively the modulation of metabolic fluxes under different conditions (Fell, 1992). The Control Coefficient is a systemic parameter related to homeostasis while the Elasticity Coefficient is a local parameter concerned with changes on the metabolic pathway. The Response Coefficient is a parameter that quantifies the effect of a modulator like a drug or hormone on a pathway but is not a component of the pathway. The coefficients are linked by the summation and inter-connectivity theorems which together make it possible to express one coefficient in terms of the others (Fell, 1992, Box 1). This essay does not consider how these parameters might be applied to analyzing the dynamics of the life cycle of a parasite. Rather the essay focuses on the main conclusions of the MCA that respectively debunk the rate limiting hypothesis and affirms the participation of multiple steps in controlling the flux of a metabolic pathway at steady state.

\subsection{The parasite's life cycle segment compared to a metabolic pathway}

It can be assumed for the purpose of the analogy that the infective stage of a parasite is the source, the different intermediary stages that occur in the immunocompetent host are the metabolites and the transmissible stage, the sink. In a generalized form the parasite life cycle in a definitive host may be depicted as follows:

$$
\begin{aligned}
& S_{i} \stackrel{E_{1}}{\longrightarrow} S_{1} \stackrel{E_{2}}{\longrightarrow} S_{2} \stackrel{E_{3}}{\longrightarrow} S_{3} \ldots \ldots S_{t} \\
& \text { where } S_{i}=\text { the infective stage } \\
& S_{1}, S_{2}, S_{3,} \ldots=\text { intermediate stage } \\
& S_{t}=\text { the transmissible stage } \\
& E_{1}, E_{2}, E_{3}, \cdots=\text { morphogenesis catalysing enzymes }
\end{aligned}
$$

The main parameters of the MCA can be adapted to the parasite life cycle segment as shown below.

$$
\begin{aligned}
& \text { Box } 2 \\
& \text { Flux }(J)=\frac{d S_{t}}{d t} \\
& \text { Flux control coefficient : } C_{E_{2}}^{J}=\frac{\partial E_{3}}{\partial E_{2}} \\
& \text { Concentration control coefficient : } C_{E_{2}}^{S_{1}}=\frac{\partial S_{1}}{\partial E_{2}} \cdot \frac{V_{E_{2}}}{S_{1}}=\frac{\partial \ln S_{1}}{\partial \ln V_{E_{2}}} \\
& \text { Elasticity coefficient : } \varepsilon_{S_{1}}^{E_{2}}=\frac{\partial V_{E_{2}}}{\partial S_{1}} \cdot \frac{S_{1}}{V_{E_{2}}}=\frac{\partial \ln \left|V_{E_{2}}\right|}{\partial \ln S_{1}} \\
& \text { Response coefficient : } \boldsymbol{R}_{p}^{J}=\frac{\partial J_{E_{2}}}{\partial p} \cdot \frac{p}{\partial J_{E_{2}}}=C_{E_{1}}^{J_{E_{2}}} \cdot \mathcal{E}_{p}^{E_{1}} \\
& \text { Given that: } \\
& \quad \text { Morphogenesis catalysingenzyme } \approx \text { morphogenesis rate }= \\
& \quad \text { No. of parasites stage/ } \mu l \\
& \text { unit time }
\end{aligned}
$$

The transformation (morphogenesis) from one parasite stage to another involves complex enzymatic processes the main ones being replication and gene activation/ suppression. However, unlike the metabolic pathway the transformation (morphogenesis) is unidirectional and irreversible. This notwithstanding a metabolic pathway at a steady state can be compared to the life cycle segment provided that its net flux is a forward reaction leading to a product (i.e. the sink).

By analogy with the metabolic pathway, it will be necessary to block several, if not all the principal stages of the parasite in order to completely disrupt the life cycle. This explains why most antiparasitic sub-unit vaccines directed to only one epitope/ antigen or even one parasite stage have 
failed so far to be fully effective (see section 3 below). In the following sections evidence from our work on malaria sub-unit vaccine as well as from the literature supporting the Postulates I-III will be presented for three important parasitic infections: malaria, onchocerciasis and schistosomiasis.

\section{Estimating the minimum composition of a parasite's sub-unit vaccine}

\subsection{P. falciparum malaria}

The malaria parasite life cycle (Bruder \&al; 2010) in the intermediary host (humans) comprises at least six stages: the sporozoite, liver stage, schizont, trophozoite, merozoite, and gametocyte.( If the hypnozoite stage is added there would be seven not six stages) Assuming that one vaccine target each can be identified on every parasite, and provided that these targets are not identical, are exclusive and their effects are additive, the minimum number of vaccine targets would be six for the B-cell arm, and six for T-cell arm, making a total of twelve. In a generalized form,

$$
C_{v}=2 n
$$

where $C_{v}$ is the minimum number of vaccine targets, $n$ is the number of intermediary stages of the parasite in the immunocompetent host. How does equation-3 check out with available data?

Growth inhibition studies with specific antibodies have been used to demonstrate the relevance of an antigen as a malaria vaccine candidate. Assuming that in order for an antibody activity to be considered as significant it has to inhibit growth by at least $30 \%$ (D ent et al;2008) the cumulative effect of inhibiting all stages simultaneously would be $360 \%$ which implies that under these conditions all the parasite stages need not be blocked in order to achieve a 100\% protective effect. However, inhibitory antibody effects can vary widely (Dent et al; 2008) and cumulative effects in vivo have to be determined empirically. This type of reasoning can be applied at the level of individual parasite stages, where it might be necessary to block more than one target to achieve complete neutralization of the parasite stage in question.

O ne can imagine at least two situations in which blocking one target with a vaccine would be sufficient to eliminate the parasite. Firstly, if the vaccine is stage-specific and acts as efficiently as a stage specific drug, it could effectively eliminate the parasite by hitting just one target. However, immune evasion mechanisms and the uneven responses in a genetically diverse population of immunocompetent hosts has largely prevented this from happening. Secondly, if the same vaccine target appears on more than one parasite stage its neutralization on these stages could have the effect of multiple targeting, thereby eliminating the parasite. In these cases the minimum number of targets, $\mathrm{x}$, will be less than $2 \mathrm{n}$ but equal to or higher than unity:

$C_{v}=1 \leq x \leq 2 n$

If the parasite has only one stage that infests the definitive host, equation-4 above will still hold. However, the MVTH concerns only parasites with multiple stages in the immunocompetent host. In recent in vitro experiments it was shown that antibodies to a chimera termed UB05-09 were more effective in blocking P. faldipanumgrowth in vitro than antibodies to its constitutive components. Furthermore a combination of antibodies against UB05-09 and AMA-1 (Apical Membrane antigen) achieved 100\% inhibition of P. faldipanm growth in culture (Dinga, JN \& Titanji, VPK, 2017).

It has been shown in human and animal experiments that irradiated sporozoites of P.faldiparum can induce complete sterilizing immunity, even though this is not long lasting (Hoffman et al. 2002). However, the RTS,S vaccine based on a single molecule (the circumsporozoite protein, CSP ) has so far attained only 30-60 \% protection (Boes \&al, 2015 ). This 
is the same for virtually all other malaria sub-unit vaccines that have reached clinical trials. They too have not performed as well as the irradiated sporozoite vaccine (Boes $\notin \mathrm{al}, 2015)$.

The multi-component malaria vaccines tested so far have been more effective than single component vaccines (D oolan \& al; 1996; Boes $\&$ $\mathrm{al} ; 2015)$ and the efficacy seems to be the greater, the greater the number of stages targeted. The cited findings support Postulates I and II above. Similar trends have been found for onchocerciasis and schistosomiasis for which extensive vaccine development studies have also been done (see below sections $3.2 \& 3.3$ ).

\subsection{Onchocerciasis}

Onchocerciasis or River Blindness is an insect borne disease caused by the filarial nematode Onchocera vdulus The life cycle of $\mathrm{O}$. vdulus comprises at least four stages that develop in the human host, namely, the infective larva (L3) the fourth larval stage (L4) and the adult stage (L5) which exhibits sexual dimorphism and the microfilariae (Mf) [Makepeace, $\mathbb{e}$ al; 2015]. By analogy with a metabolic pathway, L3 represents the source, L4 and L5 the intermediary stages and Mf, the sink.

Following equation-3 presented in section 3.1 the minimum number of sub-unit onchocerciasis vaccine targets, $C_{v}=2 n=8$, where the number of intermediary stages, $\mathrm{n}=4$.

Considerable efforts have been deployed to develop a sub-unit vaccine against O.volulus and several vaccine targets have been identified that are in the pipeline for development (Makepeace, 2015; Taylor et al, 2016). A recent review showed that the vaccine targets that induce protection in experimental systems can be found on all stages of the parasite. However, they usually were tested individually, and none of them taken alone could induce $100 \%$ protection in experimental animals. The combination of two components in one vaccine formulation produced greater protection in experimental cattle than the components taken singly (Makepeace $\&$ al; 2015). It remains to be seen whether increasing the number of vaccine targets and worm stages targeted will produce a complete protective effect in vivo.

The Mf has been considered traditionally to be a pathogenic stage and thus unsuitable for development of a vaccine (Makepeace $\notin$ al 2015). However the use of ivermectin (Mectizan) a microfilaricidal drug in decades of mass chemotherapeutic campaigns has demonstrated that Mf can be safely killed in patients provided they are not co-infected with the filarial worm Lœ loa(Basanez et al; 2008) Thus the aprior exclusion of Mf from as a vaccine target appears to be unjustified.

A further complicating factor is the endosymbiosis of Wolbachia with O.vdudus(Hoerauf \& al;2001). It has been demonstrated that the elimination of Wolbachia by treatment with doxycycline leads to the death of L5 or adult stages of O.volvulus. This observation can be exploited in designing protocols for vaccination against O.volulus infection eg by combining treatment with vaccination, or developing and applying a co-vaccine aginst O.vodulus and Wolbachia.

The MVTH postulates that both arms of the immune response should be targeted in vaccine development. Most vaccine research has focused on the humoral immune response (which is easier to probe) almost to the exclusion of the cellular arm (which is more difficult to assay). Early investigations revealed that the cellular immune plays an important role in acquired immunity to O.volvulus and this should be taken into consideration in designing vaccines aginst the parasite (Greene,1992 ).

\subsection{Schistosomiasis}

Schistosomiasis is second only to malaria among the human parasitic infections in terms of its 
morbidity and negative socio-economic impacts. Four principal species of Schistosoma account for human schistosomiasis, namely, Schistoscma mansoni, S. hematobium S intercalatum and S. japoniam The parasites have a complex life cycle that involves snails as intermediary hosts ( McManus \& al, 2008; Tebeje e al; 2016)

Following the model given in 3.1 above the life cycle stages that occur in humans are: the cercariae (the infective stage representing the source) the schistosomula, the adults (male and female) and the eggs (representing the sink) which are released into the environment through feces or urine. With four life cycle stages infecting the definitive host and in accordance with equation3 , the number of subunit vaccine components is given by

$C_{v}=2 n \Rightarrow 2 \times 4=8$.

Extensive evidence has been gathered over the years showing that it is feasible to construct a sub-unit vaccine against schistosomiasis. O ver 100 antigens have been identified and evaluated in animal models (principally mice and nonhuman primates) as candidate vaccines for schistosomiasis. Various degrees of protection, measured by the reduction of worm burden, egg out-put and/ or reduction of eggs in the tissues, have been demonstrated in vaccination-challenge experiments. From the large body of evidence analyzed (McManus \& Loukas, 2008; Tebeje et al, 2016) it is observed that:

a) The level of protection induced by single antigens hardly ever attains $90 \%$; however, when tested in combination the subunit vaccines have performed better than individual constituents.

b) Cross-vaccination is possible among the four species of schistosomes that infect humans, thereby making the construction of a common vaccine cocktail an attractive possibility.

c) Although more than a hundred vaccine targets have been identified, only about three of them have so far reached clinical trials. These three components, SM 14, Sm-TSP- and Sm 29) have so far been tested separately; it remains to be seen, if according to the MVTH hypothesis presented herein, testing them together would yield better results.

d) The overwhelming majority of the vaccine targets studied are derived from the adult worms while the schistosomulae, the eggs and the cercariae are lagging behind in this respect. In accordance with Postulates I \& II, the subunit vaccine should ideally be composed of components from all the main parasite stages since targeting just one parasite stage has proved insufficient. Multiple sub-unit vaccines need to be tested for schistosomiasis as well.

e) Both B and $\mathrm{T}$ cell arms are involved in conferring immunity to schistosomiasis. In constructing a sub-unit vaccines this should also be taken into account (Postulate III)

\section{Conclusions}

This paper presents a rational approach for estimating the minimum number of vaccine targets (epitopes / antigens) necessary to construct a highly efficacious (greater than 90 $\%$ ) sub-unit anti-parasite vaccine. D rawing from the Metabolic Control Analysis and immunological reasoning a multiple target vaccine hypothesis (MVTH) is proposed, according to which the minimum number of subunit vaccine targets is greater than one, but equal to or less than twice the number of major parasite stages occurring in the definitive host. $D$ ata reported in the literature are consistent with the MVTH at least in so far as malaria, onchocerciasis and schistosomiasis are concerned.

\section{Acknowledgements.}

The authors thank the University of Buea and the Cameroon Christian University Institute for institutional support. VPKT was responsible for deriving the equations and drafting the manuscript, and DJN and RBN for the 
preliminary experiments. We thank Dr Wilson Sama Titanji for typing the equations on computer. All authors have read and approved the final manuscript. The authors declare that they have no conflict of interest.

\section{References}

Bruder, J.T; Angov, E; Limbach, K.J; and Richie, T,L. (2010). Molecular vaccines for malaria. Human Vaccines 6(1):54-77.

Basanez, M.G., S.D. Pion, E. Boakes, J.A. Filipe, T.S. Churcher, and M. Boussinesq. (2008). Effect of single-dose ivermectin on Onchocerca volvulus: a systematic review and meta-analysis. Lancet Infect Dis 8:310-322.

Boes,A;Spiegel,H; Voepel,N; Edgue,G;Beiss,V; $\mathrm{K}$ apelski et al. (2015). Analysis of a Multicomponent, Multi-stage Malaria vaccine candidate-Tackling the cocktail challenge. PloS ONE, 10(7): e0131456 doi10 1371/ journal.pone.0131456

Dent,A.E; Bergmann-Leitner,E.S; Wilson,D.W; Tish,D.J; Kimmel R, et al.(2008). Antibodymediated growth inhibition of Plasmodium falciparum. Relationship to age and protection from parasitaemia in Kenyan children and adults. PLoS ONE 3(10) e3557.doi10. 1371/ journal.pone. 0003557.

Dinga, J.N. and Titanji, V.P.K. The use of orthologous chimera UB05-09 to probe protective immunity against malaria and East Coast fever. In: Book of Abstracts from ImmunoG ambia 2016, $19^{\text {th }}-26^{\text {th }}$ November 2016; Banjul, The Gambia. Poster \# 9.

Doolan,D.L; Sedagah,M; Hedstrom, R.C; Hobart,P; Charoenvit,Y and Hoffman,S.L. (1996). Circumventing genetic restriction of protection against malaria with multigene DNA immunization: CD 4+ cell-, interferon gamma and nitric oxide immunity. Journal of Experimental Medicine,186: 1739-1746.

D raper,S.J;Angov,E;Honi,Toshihiro;Miller,H.L; Srinivasan,P;Theisen,M and Biswas ,S. (2015). Recent Advances in recombinant protein-based malaria vaccines. Vaccine,33 (52):7433-7443

Fell, D.A. (1992). Metabolic control analysis: a control of its theoretical and experimental development. Biochemical J, 286:313-320.

Fell, D. (1997). Understanding the Control of Metabolism. Portland Press Ltd, London, pp 85191

Liao,J.C. and Delgado, J. (1993). Advances in Metabolic Control Analysis. Biotechnol. Prog; 9:221-233.

Greene,BM.(1992).Celluar and humoral immune responses in onchcerciasis. J Inf Dis;165(6):11611162

Hoffman,SL; Goh,LML; Luke,TC; Schneider,I; Le,TP; D oolan,D; Saaci,J; Vega,P; D owler,M; Paul,C; Gordon,D M; Stoute,JA; Church,LWP; Sedegah,M;Heppnar,D G ;Ballou,WR\&Richie TL.(2002).Protection of humans against malaria by immunization with radiation-attenuated Plasmodium falciparum sporozoites. J Infect Dis; 185 (8): 1155-1164.

Hoerauf H, Mand S, Adjei O, et al. (2001). Depletion of Wolbachia endobacteria in Onchocerca volvulus by doxycycline and microfilaridermia after ivermectin treatment. Lancet,357:1415-6.

Makepeace, B.L.; Babyan,S; Lustigman,S and Taylor, D.W. (2015). The case for vaccine development in the strategy to eradicate river 
blindness. Expert Rev. Vaccines,1-3 (early online)doi10.1586/ 14760584.2015.1059281

McManus, D.P. and Loukas,A .(2008). Current status of Vaccines for schistosomiasis. Clin. Microbiol. Rev. 21(1):225-242

Tebeje,B.M; Harvie, M; You, H; Loukas,A; and McManus, D.(2016). Schistosomiasis vaccines: where do we stand? Parasites and Vectors, 9:528553
WHO.(2016).World Health Report 2016.www.who.int

Received: 08/ 08/ 17

Accepted: 17/ 10/ 17 